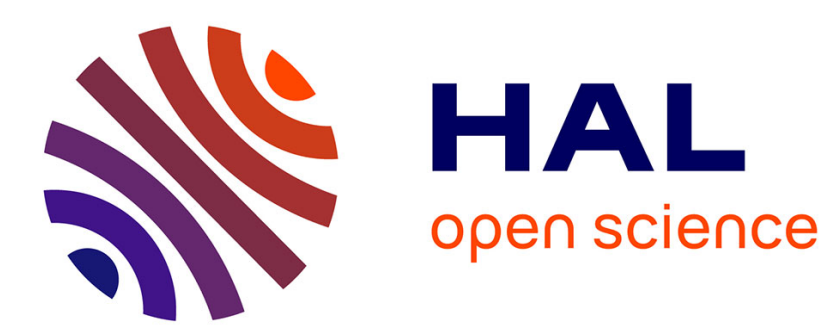

\title{
La rage en Tunisie au XIXe siècle: recrudescence ou émergence?
}

Kmar Ben Néfissa, Anne Marie Moulin, Koussay Dellagi

\section{To cite this version:}

Kmar Ben Néfissa, Anne Marie Moulin, Koussay Dellagi. La rage en Tunisie au XIXe siècle: recrudescence ou émergence?. Gesnerus, 2007, 64 (3-4), pp.173-92. 10.24894/Gesn-fr.2007.64009 . pasteur-00608463

\section{HAL Id: pasteur-00608463 https://hal-riip.archives-ouvertes.fr/pasteur-00608463}

Submitted on 13 Jul 2011

HAL is a multi-disciplinary open access archive for the deposit and dissemination of scientific research documents, whether they are published or not. The documents may come from teaching and research institutions in France or abroad, or from public or private research centers.
L'archive ouverte pluridisciplinaire $\mathbf{H A L}$, est destinée au dépôt et à la diffusion de documents scientifiques de niveau recherche, publiés ou non, émanant des établissements d'enseignement et de recherche français ou étrangers, des laboratoires publics ou privés. 


\section{La rage en Tunisie au XIX ${ }^{\mathrm{e}}$ siècle: recrudescence ou émergence?}

Kmar Ben Néfissa, Anne Marie Moulin et Koussay Dellagi

\section{Summary}

At the end of the 19th century, a canine rabies epidemics started in Tunis and in several other cities of the Beylik. Archives' data trace the epidemics back to 1870 and at that time its rapid progression was ascribed to the increase of immigration from Europe. Whether the European "street rabies virus" was also imported with the settlers' pet dogs is controversial. The epidemics might rather be linked to other factors such as socio-cultural or ecological changes. The authors try to reconstruct the history of rabies in Tunisia during this period. Changes in canine ecology and increase of dog populations in urban and suburban areas might account for the emergence of rabies at the end of 19th century and its persistence in an endemo-epidemic state.

Keywords: urban rabies; dog ecology; nineteenth century; Tunisia

\section{Résumé}

A la fin du XIX ${ }^{e}$ siècle, une épidémie de rage canine se déclare à Tunis et dans les principales villes du Beylik. Les documents d'archives situent son début à l'année 1870 et sa rapide progression apparaît associée à l'immigration croissante d'Européens. S'agit-il de l'importation du «virus des rues» européen, comme il était admis à l'époque, ou bien d'autres facteurs et déterminants écologiques, sociaux et culturels sont-ils à incriminer? Les auteurs tentent un rétro-diagnostic pour reconstituer l'histoire de la maladie en

Kmar Ben Néfissa, Institut Pasteur de Tunis, B.P. 74, Le Belvédère, TUN-1002 Tunis (qmar. benefissa@yahoo.fr).

Anne Marie Moulin, CNRS-CEDEJ, Le Caire, B.P. 392, Muhammad Farid, EG-Le Caire (moulin@cedej.org.eg).

Koussay Dellagi, Institut Pasteur de Tunis, B.P. 74, Le Belvédère, TUN-1002 Tunis (koussay. dellagi@pasteur.rns.tn). 
Tunisie. Les modifications de l'écologie canine et l'accroissement de la densité des populations canines urbaines et périurbaines pourraient expliquer l'émergence de la rage urbaine à la fin du XIX ${ }^{\mathrm{e}}$ siècle et son maintien jusqu'à nos jours à l'état endémo-épidémique.

La rage est une encéphalopathie virale mortelle transmise par morsure ou contact des muqueuses avec la salive d'un animal enragé, le chien généralement, qui est son réservoir principal. Après une incubation relativement longue quoique variable en durée, 3 semaines en moyenne mais pouvant aller jusqu'à des mois, durant laquelle le virus neurotrope gagne le système nerveux central à partir du point d'inoculation, la maladie se déclare par des troubles psychiques et comportementaux variés et inconstants suivis d'une paralysie musculaire progressive conduisant inexorablement à la mort. Décrite depuis l'Antiquité sous le terme commun d'hydrophobie, elle continue à être répandue dans le monde, sévissant sur un mode enzootique au sein de divers réservoirs mammifères et provoquant 50000 décès humains annuels (OMS) avec des dégâts importants dans le bétail malgré des méthodes de prophylaxie bien codifiées mais inégalement appliquées.

Au début du $\mathrm{XX}^{\mathrm{c}}$ siècle, en Tunisie, la rage fut considérée comme une maladie émergente avec d'autres anthropozoonoses dont le cycle de transmission inclut le chien: le kala-azar infantile méditerranéen (leishmaniose), le kyste hydatique, la fièvre boutonneuse méditerranéenne. Ces affections, que les nouvelles méthodes pasteuriennes de diagnostic scientifique permettaient de mieux cerner, étaient certainement anciennes mais se dévoilaient à la faveur de l'extension des empires coloniaux. Leur description coïncide cependant avec un changement de la conjoncture épidémiologique et sanitaire accompagnant le déclin de grands fléaux épidémiques: peste, typhus, variole, filariose, choléra. Charles Nicolle $^{1}$ estimait que l'incidence de

1 Charles Nicolle (Rouen, 1866 - Tunis, 1936), pasteurien, médecin hygiéniste et chercheur biologiste, a été directeur de l'Institut Pasteur de Tunis de 1903 à 1936 et Prix Nobel de Médecine en 1929 pour ses recherches sur le typhus (dont il démontra le mode de transmission précis et exclusif par le pou à l'homme), qui eurent une large application au moment de la Première Guerre mondiale. Ses multiples travaux de médecine expérimentale contribuèrent à identifier un certain nombre de maladies (leishmaniose, rickettsiose, fièvres méditerranéennes), de réservoirs (chien pour le kala-azar infantile, mangouste pour la rage), et à améliorer des techniques de diagnostic et de prophylaxie (sérum antirougeoleux, vaccin antityphique, vaccine). Il est aussi l'auteur d'essais philosophiques dans lesquels il développe sur le concept alors inédit d'infections inapparentes. Voir à son sujet Huet 1995. la rage humaine serait passée d'une dizaine de cas par an avant la colonisation à une centaine et plus d'individus effectivement mordus par un animal enragé et heureusement pris en charge par le service de vaccination antirabique nouvellement créé à Tunis. Il pensait aussi qu'après un maximum d'intensité entre les deux guerres mondiales, l'épidémie allait fléchir jusqu'à son plus bas niveau. Ce ne fut pas le cas (fig. 1).

Cet article s'efforce de reconstituer l'histoire de la rage en Tunisie durant la période charnière de la fin du $\mathrm{XIX}^{\mathrm{e}}$ siècle au début du $\mathrm{XX}^{\mathrm{c}}$ et s'interroge sur les facteurs ayant contribué à faire de la rage une maladie apparemment «émergente». Il est toujours délicat de pratiquer le rétro-diagnostic dans un passé plus ou moins lointain, mais la méthode historique permet néanmoins de formuler des hypothèses avec un certain degré de probabilité. A partir de certaines sources primaires, documents d'archives officiels et presse écrite, ainsi que de la littérature médicale et non médicale européenne de l'époque, nous tentons de restituer la trame chronologique d'événements médicaux majeurs, de les insérer dans leur contexte psycho-social et culturel et de discuter leur signification épidémiologique probable à travers les représentations variées que les professionnels et le populaire en avaient: la rage étaitelle une maladie d'importation comme tendent à le suggérer les documents de l'époque, ou bien était-elle la manifestation d'une nouvelle pathocénose, c'est-à-dire d'une période de l'histoire d'Afrique du Nord «marquée par un complexe pathogène particulier, régionalement différencié, formé d'un ensemble assez stable d'affections courantes» $?^{2}$

\section{Les débuts de l'épidémie: la rage, maladie importée?}

A l'instar de la plupart des pays nouvellement intégrés à l'Empire français, les premières séries statistiques épidémiologiques officielles sont apparues en Tunisie avec l'établissement du protectorat français (1881), très rapidement suivi par la création du centre antirabique (1893), futur Institut Pasteur de Tunis. Ces statistiques attestent une flambée épidémique de rage à la fin du XIX ${ }^{\mathrm{e}}$ siècle $^{3}$.

En fait, deux documents manuscrits officiels notifient un début de cycle de rage à Tunis dès 1870 . Le premier est une circulaire datée du 16 juin 1876, traduite en français et adressée par le premier ministre du Bey aux consuls européens:

2 Biraben 1985, 293-301.

3 Loir 1898. 


\section{Circulaire:}

Le 23 de Rabi el Eouel (18 avril 1876) de l'année courante, nous vous faisions part des plaintes portées contre la circulation démesurée des chiens errants en liberté dans la ville, abus qui a causé de nombreux accidents aux habitants. Dans cette circulaire, nous vous informion de porter à la connaissance de vos administrés que les agents de la police avaient ordre de s'emparer de tout chien trouvé circulant sans muselière trois jours après la date de notificaion en question.

Ces dispositions n'ont pas été suivies par les propriétaires de chiens et nous venons d'apprendre, avec beaucoup de regret, que de nouveaux accidents sont arrivés tout dernièrement et ont occasionné une certaine frayeur parmi les habitants, par suite de cas d'hydrophobie causés par ces mêmes abus de laisser circuler librement les chiens en ville.

Comme il est de notre devoir de pourvoir à la sécurité des personnes, nous nous adressons de nouveau à vous en vous priant d'informer vos administrés que dans un but d'intérêt général, l'ordre vient d'être donné aux agents de la police locale de tuer invariablement tout chien qui sera rencontré dans la rue de la ville circulant sans muselière ou sans autres moyens de sécurité.

Signé: Khéreddine Pacha. ${ }^{4}$

Et le second document est une note manuscrite signée par un vétérinaire fonctionnaire de l'Etat tunisien, français d'origine, le Dr Bouquet, datée du 12 juin 1876 et adressée au premier ministre ${ }^{5}$. On y trouve une mise au poin de l'actualité médicale de l'époque ${ }^{6}$ sur la rage et sa prophylaxie ainsi que quelques informations sur la situation épidémiologique à Tunis, avec les paragraphes suivants:

En présence de nombreux cas de rage qui se produisent à Tunis depuis quelques mois [...] Les renseignements pris à Tunis m'ont fait connaître que ce n'est que depuis cinq ans tout au plus que la rage y a fait sa première apparition, il est probable qu'elle a été importée par des chiens venant d'Europe.

L'idée que la rage est une maladie d'importation venue du Vieux Continent est très ancrée à l'époque, et elle continue à être répandue jusqu'à nos jours. Elle se fonde sur les observations épidémiologiques de la fin du XVIII ${ }^{\mathrm{e}}$ siècle qui relèvent que la maladie suit le sillage de l'expansion européenne. Des épidémies éclataient dans les villes portuaires d'Asie, d'Amérique, du Pacifique ou d'Afrique, cö̈ncidant avec le débarquement de chiens venus d'Europe: Boston et Amérique du Nord (1768) avec une épizootie chez le loup et le renard en 1810, Indes Occidentales françaises (1771), Saint Domingue, Jamaïque, Pérou (1783 et 1803), Ile Maurice (1815), Malte (1847),

4 Archives Nationales du Gouvernement de Tunis, série historique, dossier 626, carton 57, circulaires relatives à l'interdiction des chiens errants de 1860 à 1882 .

5 Archives Nationales du Gouvernement de Tunis, série historique, dossier 795, carton 66 , armoire 7, pièce 6, note du Dr Bouquet.

6 Bouley 1870.

7 Il n'y a pas lieu de douter de l'affirmation qui rapporte les premiers cas de rage dans la capitale à l'année 1870. A l'époque, les médecins européens en Tunisie sont en position privilégiée dans la cour beylicale et le réseau consulaire, ce qui leur donne accès à des informations fiables. De plus, la surveillance de la santé publique est déjà bien établie dans le cadre des politiques de quarantaine contre la peste et le choléra. Voir Gallagher 1982.
Hong Kong (1857), Shanghai (1867). En Europe, le loup avec le chien a été pendant longtemps le réservoir connu de la maladie. D'après les séries statistiques de Victor Babès ${ }^{8}$, des pics épidémiques de rage canine sont notés dans de nombreuses villes d'Europe sur un fond endémique constant: Londres (1759, 1806, 1865), Vienne (1815, 1830, 1855), Hambourg (1830, 1851-1856), Paris (1831-1836), Lyon (1841/42 et 1864/65), Bruxelles (1868). Une vague d'épizootie canine gagne tout le bassin méditerranéen (France, Italie, Espagne) en 1763, tandis qu'une épizootie chez le renard se déclare au pied des Montagnes jurassiennes en 1803. De nombreux pays adoptent une réglementation prophylactique de police sanitaire imitant le modèle de l'Allemagne prussienne (ordonnance de 1835) avec campagnes d'abattage des chiens errants, obligation de port de la muselière, taxe canine et amendes aux propriétaires de chiens délictueux. Un congrès international vétérinaire réunit les savants à Vienne en 1865 et discute de la question. Certains gouvernements instaurent une quarantaine pour les chiens importés et viennent à bout de la maladie en Australie, Grande Bretagne et Malte.

En Afrique, la rage était à la fin du $\mathrm{XIX}^{\mathrm{c}}$ siècle une maladie apparemment nouvelle sur le continent; le premier cas notifié en Afrique subsaharienne date de $1893^{\circ}$. Cependant, une maladie paralytique rapidement évolutive dite $\mathrm{du}$ «chien fou», oulo fato en dialecte local, a été décrite dès 1815 au Sénégal et, plus tard, diagnostiquée comme rage ${ }^{10}$. Elle aurait été introduite par les caravaniers du Nord de l'Afrique où des épidémies commencent à être notifiées d'abord en 1854 à Alger, sous domination française, puis à Tunis en 1876. Des foyers enzootiques sauvages dont on ne connait pas l'ancienneté seront plus tard mis en évidence chez les canidés sauvages (chacal, chien sauvage, loup d'Ethiopie) et les herpétidés (mangoustes) en Afrique centrale, septentrionale et orientale. En Egypte, Clot Bey observe au milieu du $\mathrm{XIX}^{\mathrm{c}}$ siècle que la rage est présente sur le territoire et il cite le chien mais aussi le renard, le chacal et la belette comme animaux susceptibles tout en affirmant son caractère spontané; il publie un protocole de traitement des blessures par morsures d'animaux enragés ${ }^{11}$. En Orient musulman, les observateurs du XIX ${ }^{\mathrm{c}}$ siècle notent que la rage est absente, du moins dans les villes. Le Dr Bouquet relève:

L'existence de la rage dans les pays chauds a été très longtemps niée. Les médecins attachés au service sanitaire en Egypte, en Syrie, en Turquie, affirment qu'elle était inconnue il y a sept ou huit ans. Prince, ancien directeur de l'Ecole vétérinaire d'Abouzabel (Egypte) n'y a pas vu un seul cas de rage en dix ans. ${ }^{12}$

8 Babès 1912.

9 Bingham 2005

0 Balozet 1938

1 Camussi/Chérif 1910.

12 Archives Nationales du Gouvernement de Tunis (voir note 5). 
Cette impression est confirmée plus tard par Adrien Loir ${ }^{13}$ et Paul Remlinger ${ }^{14}$, qui notent quelques rares cas de rage muette à Constantinople, sans véritable cycle, malgré la prolifération de «chiens qui n'appartiennent à personne» et «qui sont à la ville». Quelques années auparavant, le Sultan Abdulhamid avait ordonné leur déportation sur un rocher du Bosphore d'où leurs aboiements avaient ému l'Europe ${ }^{15}$.

Le contexte international du XIX ${ }^{\mathrm{e}}$ siècle est favorable à la diffusion du «virus des rues» européen. Son importation en Tunisie est tout à fait vraisemblable, par deux voies de pénétration au moins: terrestre à partir de l'Algérie ou maritime à partir des villes côtières européennes, Malte ou la Sicile par exemple. Mais cette hypothèse n'élimine pas celle de la préexistence du pathogène sur le territoire. Dès lors et en l'absence de statistiques chiffrées ou de documents officiels, il y a lieu de s'interroger sur la situation de la rage au XIX ${ }^{\mathrm{c}}$ siècle en Tunisie. Existait-elle? Etait-elle identifiée en tant que maladie? Quel était son vécu psycho-social?

\section{Médecine arabe savante et pratiques populaires au $\mathrm{XIX}^{\mathrm{e}}$ siècle: ancienneté de la rage}

La rage sévissait, selon toute vraisemblance, dans le bassin méditerranéen depuis l'antiquité gréco-romaine. Dans l'ancienne Numidie, elle serait notamment représentée sur une mosaïque datant du $\mathrm{IV}^{\mathrm{e}}$ siècle $^{16}$, sous forme d'un animal mi-chacal et mi-chien en posture agressive évocatrice et à la queue écourtée (ne dit-on pas que les bergers antiques coupaient la queue de l'animal pour le prévenir de la rage?). Son caractère épidémique fut reconnu au point de la faire considérer comme une «maladie nouvelle» par des disciples d'Hippocrate ${ }^{17}$. Elle aurait commencé à être individualisée dans les traités de médecine grecque, d'abord chez le chien uniquement, puis chez le chien et l'homme; puis d'autres animaux sont cités: renard, chacal, belette, loup, animaux domestiques, par Avicenne notamment ${ }^{18}$. Elle est désignée en grec par le terme de lyssa, puis en latin par celui de rabies, qui évoquent tous deux la fureur et les atteintes nerveuses, et en français jusqu'à la fin du XIX ${ }^{\mathrm{e}}$ siècle par le terme savant d'hydrophobie, en relation avec le symptôme 13 Loir 1896.

14 Remlinger 1903

15 Moulin 1992.

16 Coulin 1992.

17 Celse 1838 .

18 Les médecins grecs et arabes ont décrit la rage chez le chien et chez l'homme avec une symptomatologie à peu près identique, sans que I'on sache s'il s'agissait d'observations authentiques ou de reprises et commentaires de leurs prédécesseurs. Néanmoins, l'évocatio de la faune sauvage comme réservoir de la rage serait une nouveauté d'Avicenne. considéré alors comme pathognomonique de la maladie. Dans la médecine arabe classique, la maladie est évoquée sous le terme de keleb, qui pourrait être la transcription d'un terme grec, peut-être celui de kunalussa, rage du chien, et traduit initialement par hydrophobie. On y retrouve néanmoins la racine arabe de kelb, c'est-à-dire chien, retrouvée aussi dans l'adjectif mekloub, qui signifie enragé. C'est sous le terme de keleb que la rage fut décrite pour la première fois en Tunisie par le grand médecin de Kairouan Ibn Jazzar (898-980) aux chapitres des envenimations ${ }^{19}$. Il évoque, parmi les signes, «le chien qui ne reconnaît plus son maître» et les «rêves» prodromiques de la rage humaine ${ }^{20}$. La rage est alors considérée comme une espèce d'empoisonnement d'origine animale avec une explication physiopathologique se référant aux paradigmes de l'époque: un excès de bile noire ou atrabile chez le chien dont le tempérament est naturellement sec et froid. Les causes de la maladie sont soit internes (pourrissement de l'atrabile), soit externes, effets des saisons et des températures extérieures extrêmes (trop chaudes ou trop froides) ou de l'ingestion par le chien d'aliments avariés et putréfiés. L'apparition de l'hydrophobie, folie d'origine atrabilaire, est un signe fatal: certains auteurs anciens recommandent d'obliger le malade à boire avec un entonnoir après l'avoir attaché ou bien de le plonger dans l'eau en lui maintenant la tête sous l'eau, et ... abréger ainsi ses souffrances. Des insectes de l'ordre des coléoptères (cantharide, cétoine) ont été prônés par des médecins arabes médiévaux comme l'antidote spécifique de la rage ${ }^{21}$.

19 Abou Jaafar Ahmed Ibn Ibrahim Ali Ibn El Jazzar est le plus célèbre des médecins de l'école de Kairouan pour ses nombreux traités de médecine et de pharmacie, dont le plus fameux est Zad el mousafir, ou Provisions du voyageur connu également sous le titre de Viatique. Cet ouvrage fut traduit dès le $\mathrm{X}^{\mathrm{c}}$ siècle, de son vivant dit-on, par Constantin l'Africain de l'école de Salerne sous le titre de Viaticum perigrinantis, puis en grec au XI' siècle sous le titre d'Ephodes, puis au XIII'siècle en hébreu sous le titre de Tzadad Deracim; au XVI'siècle, il connut une large diffusion en Europe avec quatre éditions imprimées sous différents noms d'auteurs. L'auteur véritable, Ibn Jazzar, ne sera révélé à l'Europe qu'en 1851 grâce aux recherches effectués par Charles Daremberg sur une copie manuscrite arabe déposée à Dresde et qu'il fit traduire en français (y compris le chapitre sur la rage) par Gustave Dugat et publier dans le Journal Asiatique d'avril-mai 1853. Mais ses ouvrages étaient connus, et publier dans le Joumal Asiatique davril-mai 1853. Mais ses ouvrages étaient connus, commentés et cités par les médecins arabes d' Andalousie et d' Orient. Voir l'ouvrage en arabe d'Ibrahim Ben Mrad, Recherches sur l'histoire de la médecine et de la pharmacologie chez les
Arabes (Dar Al-Gharb Al-Islami, Beyrouth 1991). Voir aussi Ammar 1997.

20 Ibn Jazzar 1999, tome 2, partie 7 sur les affections de la peau, chapitre 13 sur le keleb:638-642, réf. 15.

$21 \mathrm{La}$ cantharide, dont on extrait aujourd'hui la cantharidine douce, est diversement citée par les auteurs grecs: tantôt comme médicament caustique, tantôt comme poison avec son antidote, tantôt comme ingrédient du traitement de diverses affections cutanées ou de l'appareil génito-urinaire. Elle avait aussi la réputation d'être aphrodisiaque à faible dose. Dans la rage, elle a été prescrite par Avicenne (980-1036) en deux prises orales quelques jours après la morsure, avec la particularité d'induire l'émission de sang dans les urines accompagnée d'atroces douleurs abdominales, signe de guérison (par évacuation du poison?): Camussi/Chérif 1910. 
La rage semait la terreur dans les communautés exposées et bien des méthodes empiriques et mystico-magiques de prévention sont apparues au fil du temps dans les différentes régions du globe. Quelle était la situation de la rage en Tunisie au cours de la période précoloniale? On ne retrouve pas de trace écrite de continuité de la maladie entre le $\mathrm{X}^{\mathrm{e}}$ et le XVIII ${ }^{\mathrm{e}}$ siècle jusqu'au témoignage du médecin botaniste français, Jean André Peysonnel qui, en voyage d'études en Tunisie en 1724, traverse le village de Grombalia au Cap Bon, à une trentaine de kilomètres de Tunis. Il note:

Il y là un puits d'eau qui n'est pas bonne à boire; mais elle a une vertu spéciale contre la rage lorsqu'on s'y baigne ou qu'on en boit. L'expérience, dit-on, prouve que toutes les personnes et les animaux enragés ou qui ont été mordus par les animaux attaqués de l'hydrophobie, ont été guéris ou garantis lorsqu'on en a soin de les baigner dans l'eau de ce puits. Cette année même, nous dit-on, cinq personnes du lieu de Soliman ont été mordues d'un chien enragé, quatre furent se baigner dans cette eau et le cinquième n'y fut pas, parce que le chien disaitelle, ne lui avait mordu que la robe. Avant les quarante jours, elle fut attaquée de rage et mourut; les autres furent préservées. Les Maures attribuent la vertu de cette eau aux mérites d’un marabout qui est enterré près de là. ${ }^{22}$

Il s'agit du lieu dit Aïn Zlazlyya près du mausolée de Sidi Bouzikri au sudouest de Grombalia ${ }^{23}$. Mais il existait d'autres points d'eau sacrés réputés pour leur vertu antirabique, tels que Bir el afou (puits du pardon) près du mausolée de la zaouia de Sidi Abdelkader El Jilani au Cap Bon, Oued El Meselmane et son embouchure appelée Bahr El Selmin (la mer des sauvés) près de Mahdia dans le Sahel, Gueltet ech-cheffa (étang de la guérison) dans le Djerid au sud, ou bien un puits près de la zaouia Sidi Daoud dans la banlieue nord de Tunis, ou encore la mer faisant face au mausolée de Sidi Mansour près de $\mathrm{Sfax}^{24}$.

D'autres pratiques populaires contre les morsures animales suspectes sont aussi répandues. Ainsi, entre 1886 et 1900, des thérapeutes traditionnels installés dans diverses villes étaient connus comme spécialisés dans les morsures d'animaux enragés, fréquentés avec un certain degré de confiance par la population y compris les Européens ${ }^{25}$. Leur compétence commune résidait

22 Peysonnel 2003, 114sq.

23 Curieusement, ce mausolée est situé près d'un domaine agricole renfermant des vestiges antiques appelé Henchir el Kalbyya, qui pourrait être traduit par «chenil» ou «lieu à forte concentration de chiens»: Peysonnel 2003.

$24 \mathrm{Au}$ moment du débat public sur la mise au point par Pasteur du vaccin contre la rage, des études sur les coutumes antirabiques en Afrique du Nord furent publiées dans divers journaux scientifici Asiatique en 1888, puis inté Asian ration du Dr Ahmed Cherif, troisieme médecin musulman tunisien

25 Archives Nationales du Gouvernement de Tunis, Série E-620-5/9, pièce 19. Cas d'une jeune Anglo-Maltaise de Zarzis mordue en 1900 par un chien enragé, qui préfère consulter un tradipraticien de Djerba plutôt que se faire vacciner. dans la prescription de coléoptères, la cantharide en particulier appellée $d z e r$ nouh en arabe ou dzerarih au pluriel. Les techniques de collecte de l'insecte, le choix des variétés, sa préparation et son stockage, son dosage et les modes de prise par le malade révèlent un savoir-faire ancien et une expérience du produit et de la maladie ainsi que de sa durée d'incubation. L'émission de grumeaux de sang dans les urines, appelés «petits chiens», est considérée comme un signe de guérison. Par ailleurs, le dzernouh est vendu par les apothicaires des souks sans que l'on sache quel usage les gens en faisaient. Ahmed Chérif note que la consommation de ce produit antirabique et supposé abortif se répand à partir du $\mathrm{XVII}^{\mathrm{e}}$ siècle dans certaines contrées d'Europe et de Russie, au rythme, selon lui, de la diffusion des versions traduites et imprimées des traités arabes ${ }^{26}$. Parmi les autres pratiques populaires, citons l'usage d'une plante de la famille des labiées, appelée chedengoura, la consommation du foie ou du cœur du chien mordeur ou du lait de femme, la plongée du membre blessé dans les viscères fumants d'un mouton que l'on vient de sacrifier. Le traitement local se faisait selon les recettes anciennes de la médecine grecque et arabe, avec cautérisation au fer rouge de la plaie de la morsure qui n'était pas suturée et/ou application de cantharides et d'emplâtres de diverses compositions.

L'hydrophobie est un symptôme reconnu par les nomades. Le chien ou l'homme atteint de la rage est isolé et parfois aspergé ou plongé dans l'eau, conduites retrouvées aussi dans certaines régions d'Europe. Des rituels mystico- magiques à visée prophylactique sont aussi rapportés tels que le port d'amulettes sur lesquelles des versets du Coran sont écrits par des descendants de lignées maraboutiques dont l'ancêtre est réputé protéger de la maladie, ou le port de dents d'animaux enragés. Ces pratiques populaires, manifestement inspirées des traités de médecine ancienne, révèlent que les Arabes au $\mathrm{XIX}^{\mathrm{e}}$ siècle connaissaient la rage, son mode de transmission et sa durée d'incubation, ou du moins que la morsure animale y était redoutée.

Une fois l'hypothèse de la présence ancienne de la rage admise comme hautement probable, il reste à supputer sa fréquence et ses modalités épidémiologiques, afin de rendre compte de la «recrudescence» observée à la fin du XIX ${ }^{\mathrm{c}}$ siècle et confirmée par les statistiques. 


\section{Les transformations socio-culturelles de la fin du XIX ${ }^{\mathrm{e}}$ siècle:} émergence de la rage urbaine

Avec la naissance de la médecine moderne, on assiste à une vision nouvelle de l'épidémiologie de la maladie. Vers 1870, des chercheurs allemands et français démontrent la virulence de la salive et du système nerveux à partir de cadavres d'animaux enragés, mettant fin au débat sur l'origine spontanée ou contagieuse de la maladie et ouvrant la voie à de nouvelles méthodes de diagnostic telles que la visualisation des corps de Negri dans les cornes d'Ammon ou l'induction de rage expérimentale sur des lapins, par inoculation intracérébrale du bulbe d'animaux enragés ${ }^{27}$. Cette découverte a permis aussi de définir la période d'excrétion salivaire qui est celle où l'animal peut transmettre la rage: elle précède d'un à deux jours les premiers signes cliniques, dure une semaine en moyenne. Cela détermine un indice épidémiologique important qui est le taux de transmission de base, c'est-à-dire le nombre moyen d'hôtes indemnes contaminés par un animal enragé durant toute sa phase infectieuse ${ }^{28}$, qui rend compte de la tendance évolutive d'une infection dans un lieu donné et dans une période donnée: tendance épidémique (indice $>1$ ), tendance endémique (indice $\geqq 1$ ) et tendance à l'extinction (indice $<1$ ). Pour la rage, il est étroitement lié au nombre de contacts entre l'animal enragé et les hôtes susceptibles et dépend donc de la densité canine. La disponibilité de nourriture et de gîtes pour l'élevage des jeunes chiots par la mère est un facteur d'accroissement ou de maintien du régime démographique de la population canine. De ce fait, l'importance de la population canine, la nature et l'intensité de la relation entre l'homme et le chien, animal domestique par excellence, sont les deux facteurs clés de l'épidémiologie de la rage ${ }^{29}$.

La littérature médicale et scientifique du début du $\mathrm{XX}^{\mathrm{e}}$ siècle a tendance à comparer la relation chien-homme des Européens à celle des autochtones. Ainsi Adrien Loir, premier directeur de l'Institut Pasteur de Tunis de 1893 à 1902, note la présence de chiens qui circulent librement dans la ville de Tunis, mais pour lui, la situation est comparable à celle des villes européennes de l'époque et la rage n'y est pas plus répandue et ce sont les «chiens arabes absolument libres dans les campagnes et sur lesquels il est impossible d'exercer une surveillance» qui entretiennent l'épidémie ${ }^{30}$. Quelques années plus tard, en 1914, Charles Nicolle, son successeur, estime pour sa part que la

27 Organisation d'Hygiène de la Société des Nations 1927.

28 Bingham 2005

29 Wandeler/Capt 1985

30 Loir 1896. rage est «infiniment trop fréquente en Tunisie» et désigne inlassablement les propriétaires de chiens européens qui, par leur cynophilie et leur «incurie» ${ }^{31}$, sont responsables de la situation. Les mesures des pouvoirs publics contre la rage ont rencontré une vive résistance dans les communautés européennes de Tunisie et ont été inégalement suivies: dans le quartier dit «La Petite Sicile» près du port de la Goulette, par exemple, les chiens errants étaient cachés pour les soustraire à la capture par les camions de la fourrière; les fermiers européens de Bizerte refusaient de déclarer les morsures animales suspectes et de se séparer de leur chien même en cas d'exposition. A l'inverse, le paysan musulman ne rechignait pas à abattre la bête ainsi que tous les animauxcontacts, y compris les volailles!

Cette différence dans les attitudes était une réalité sociale perceptible à l'époque et trouve son origine dans des croyances et coutumes religieuses vivaces jusqu'à nos jours. En effet, les relations traditionnelles entre les habitants de l'Afrique du Nord et le chien n'ont jamais été chaleureuses et encore moins affectueuses. L'animal est considéré comme impur et il est proscrit par les courants doctrinaires majoritaires des religions musulmane et juive. Dans le rite malékite, en vigueur en Tunisie depuis le $\mathrm{IX}^{\mathrm{e}}$ siècle, sa domestication est formellement interdite sauf pour deux catégories de gens: les bergers pour la surveillance et l'accompagnement des troupeaux et le chasseur lorsque la chasse vise à nourrir ou à protéger la communauté et ne poursuit pas des buts ludiques ${ }^{32}$. Ces croyances sont défavorables à l'animal: les citadins éprouvent de la répugnance à son égard, il ne rentre pas dans les maisons, il n'est pas touché et il est même chassé. Il n'était pas admis dans les médinas, vieilles cités orientales fortifiées, protégées par de hautes murailles avec des portes qui sont fermées la nuit. Ainsi cette scène insolite dont fut témoin Alexandre Dumas, écrivain et médecin français, à son débarquement en 1846 sur les berges du Lac de Tunis:

Cette jetée était couverte de charpente et de matériaux de construction. Avec la nuit, tombant rapide, nous apparut un des caractères distinctifs des villes d'Orient. Devant nous, derrière nous, les chiens commençaient à se rassembler; chiens hideux et qui n'obéissaient à aucun maître, dont l'aspect sauvage tient à la fois du renard et du loup qui hérissent leur poil, roidissant leur queue et hurlent aux passants. Ces chiens en troupe nous suivaient, comme curieux de voir des étrangers. Un, entre autres, monté sur le faîte d'un long mur, nous

31 Nicolle 1916; Nicolle 1920.

32 Le malékisme est l'une des quatre écoles de jurisprudence de la religion musulmane. Outre le Coran et la Sunna (ensemble de traditions inspirés par les paroles et les hauts faits du prophète Mohamed), cette école se fonde sur les coutumes des habitants de la ville sainte de Médine comme source de jurisprudence: voir Ibn Abi Zyad El Kairaouani 1961. L'Imam Sahnoun (777-854), fondateur du malékisme en Ifrikyia, fut qadhi (chef de la justice de la ville) de Kairouan après son retour des lieux saints et aurait ordonné, en cette qualité, à la police d'abattre tous les chiens de la cité: voir l'ouvrage en arabe du Dr Ahmed Ben Miled, La médecine arabe tunisienne (Tunis 1987) 201. 
accompagnait en aboyant, faisant mine à chaque instant de vouloir plonger sur nous [...]. Arrivés aux portes, ils nous quittèrent. ${ }^{33}$

Quant aux communautés rurales et pastorales qui apprivoisent, voire dressent au besoin l'animal pour la surveillance, elles sont éparpillées géographiquement et résident dans des tentes ou des gourbis, à proximité des forêts du nord et des côtes, des oasis dans le sud et elles se déplacent au gré des besoins du commerce, de la transhumance et du marché saisonnier de l'emploi agricole. Ce mode de vie instable est défavorable à la reproduction canine. La mobilité de ces communautés assure néanmoins la pérennisation de la maladie à grande échelle en créant des occasions de contacts entre le chien et la faune sauvage et entre le chien enragé et d'autres populations canines locales. Des cycles enzootiques sont concevables: le chacal est cité autant que le chien comme animal mordeur enragé en Afrique du Nord au XIX ${ }^{\mathrm{e}}$ siècle et figure régulièrement dans les statistiques épidémiologiques de la rage jusqu'en $1970^{34}$. La mangouste, en tant que réservoir, a été découverte et décrite pour la première fois dans la littérature médicale par Charles Nicolle, au nord de la Tunisie, du côté de la frontière avec l'Algérie, en $1903^{35}$.

Il existe des exceptions à ce schéma: certains groupes dans le sud, à Gabès ou à Sfax par exemple, consomment de la viande de chien pour ses vertus thérapeutiques réputées contre le manque d'appétit, la syphilis, la phtisie, les fièvres, et font de l'élevage ${ }^{36}$ avec un marché pour chiens dans certaines villes. Ces pratiques sont cependant cachées et sont de possibles survivances de l'époque antéislamique, lorsque la chasse et son élevage faisaient partie des usages de la bonne société comme en témoignent de nombreuses mosaïques.

Le rejet du chien par les citadins et son relatif abandon par les gens de la campagne justifient l'hypothèse d'une densité canine faible jusqu'au $\mathrm{XIX}^{\mathrm{e}}$ siècle. L'animal vit dans un état semi sauvage, commensal de groupes nomades et tribaux et, dans tous les cas, hors des cités. La rage sévissait selon toute vraisemblance sur un mode endémo-sporadique dans les campagnes mais restait suffisamment répandue pour justifier la survie de pratiques populaires antirabiques et la transmission orale de méthodes de prophylaxie. Aujourd'hui, on parlerait d'état endémique avec un taux de transmission de base proche de 1 . Dès lors comment expliquer la flambée épidémique de la fin du XIX ${ }^{\mathrm{c}}$ siècle et sa persistance jusqu'à nos jours?

33 Dumas $1982,30$.

34 Voir les statistiques d'activités du service de la rage de l'Institut Pasteur de Tunis, publiées régulièrement dans ses Archives, particulièrement les années de 1906 à 1915.

35 Nicolle/Chaltier 1904

36 Bertholon 1896
La recrudescence de la rage coïncide en fait avec son intrusion dans le tissu urbain. Ce constat amène à s'interroger sur les modifications de l'épidémiologie urbaine et sur la situation nouvelle induite par l'arrivée massive d'Européens: la population italienne de Tunisie, estimée en 1858 à 5000 individus, passe en 1901 à 71000 individus ${ }^{37}$ pour une population totale d'environ 2 millions d'habitants. Les minorités européennes et chrétiennes vivent dans les villes dont elles représentent près de 5 à $10 \%$ des habitants. Les historiens décrivent une Tunisie de la fin du XIX ${ }^{\mathrm{c}}$ siècle en proie à de profondes mutations économiques, sociales et démographiques ${ }^{38}$. La naissance de l'organisation municipale et la création par décret beylical en 1858 de la municipalité de Tunis s'accompagnent de nouvelles mesures «à l'européenne» d'hygiène collective ${ }^{39}$ telles que le ramassage des ordures, avec comme corollaire la multiplication de décharges publiques qui vont créer des sites nourriciers stables pour les canidés. La plus importante est située sur les berges du Lac de Tunis qui a de tout temps servi de déversoir aux égouts et déchets de la ville; mais au sein même de la médina, dans chaque quartier, de nouveaux dépôts d'ordures sont aménagés. Le fait le plus marquant de cette période reste cependant la naissance et le développement rapide de la nouvelle ville européenne sur la jetée du lac, là où précisément, en 1846 , Alexandre Dumas ${ }^{40}$ en décrit les prémices, en signalant un chantier de construction. C'est sur «le terrain vague» qui mène à la porte orientale de la ville appelée Bab Bhar, porte de la mer, que les immeubles d'architecture européenne, les échoppes, les hangars et les baraquements sont peu à peu érigés, témoignant de la montée en puissance des minorités européennes très actives et présentes dans toutes les branches de l'activité économique. Cette urbanisation ne cessera ensuite de s'étendre et de gagner sur les terres environnantes, créant à chaque extension une ceinture suburbaine, absorbant chaque fois d'anciens agglomérats ruraux, attirant sur ses franges excentrées les chiens errants. En 1898, un demi-siècle après la visite de l'écrivain, les bêtes «hideuses», agressives, aux habitudes nocturnes ont disparu et sont remplacées par une gent canine familière, aux habitudes diurnes ${ }^{41}$. Fait encore plus remarquable, les chiens ont franchi le «long mur» car les quartiers européens et traditionnels s'ouvrent les uns aux autres, une partie des murailles de la vieille médina est détruite vers 1860 et les fameuses portes médiévales, dont deux sont démolies, ne sont plus fermées la nuit ${ }^{42}$. Il

37 Romain 1999

38 Valensi 1977

39 Chabbi 1977.

41 Archives Nationales du Gouvernement de Tunis (voir note 25); Loir 1896.

42 Sebag 1989. 
n'y a plus de frontière étanche entre le cœur du vieux Tunis, les nouveaux quartiers européens, les faubourgs et les campagnes environnantes. E les échanges deviennent incontrôlables. Les chiens circulent librement d'un espace à l'autre, ils prennent de nouvelles habitudes consolidant leur fonction d'éboueur. Les nouveaux migrants européens importent leurs coutumes, en particulier celle de l'animal de compagnie et de l'intimité avec le chien. De nouvelles races canines européennes apparaissent (bergers allemands, caniches, épagneuls ...) et croisent avec la race kabyle locale ou «chien arabe» ${ }^{43}$.

Le paysage rural change aussi. Des travaux multiples de mise en valeur agricole sont entamés à partir du milieu du XIX ${ }^{\mathrm{e}}$ siècle $^{44}$ : construction de routes, de chemins de fer, de télégraphes et modifient les frontières ancestrales entre périmètres forestiers, pastoraux et de plantation, introduisant des changements dans la biodiversité. Le développement de l'agriculture à caractère commercial, de même que l'intensification de la chasse, s'est accompagné d'une modification durable de l'écologie animale traditionnelle, avec disparition d'une partie de la faune sauvage résiduelle. Le dernier lion est abattu en 1898 dans la région de Tabarka, au nord du pays. La sédentarisation des nomades, sous l'effet de mauvaises conditions économiques et sanitaires (famine et typhus de 1868 et 1873 entre autres) ${ }^{45}$ et le regroupement des agglomérations rurales sont autant de facteurs favorables à la migration des chiens vers les douars, groupements d'habitats ruraux, puis vers les décherats, villages ruraux, et, de là, vers les ceintures suburbaines des villes plus importantes. Les relevés épidémiologiques des premières années de fonctionnement du service antirabique de l'Institut Pasteur de Tunis révèlent peut-être ce «brouillage» des paysages limitrophes ruraux et urbains traditionnels: une grande diversité d'espèces animales diagnostiquées enragées est retrouvée de manière constante, aussi bien domestiques (chats, souris, chèvres, moutons, vaches, chevaux, ânes, dromadaires) que sauvages (renard, chacal, mangouste, belette, hyène) révélant peut-être de nouveaux contacts entre ces espèces, la migration de certaines d'entre elles ou la mise à découvert de foyers enzootiques jusque là invisibles (particulièrement dans le Sahel: Grombalia et Sousse $)^{46}$. Ainsi, un réservoir principal remplace l'autre, sous la pression des facteurs environnementaux et/ou de contrôle de la maladie: le chien a remplacé le loup ou le chacal quand ces espèces se sont

43 Voir rubriques «police des chiens» et «chiens trouvés», dans les journaux L'Echo de Tunis (dernier trimestre 1888 et 1889) et La Dépêche Tunisienne (janvier 1892). Dans le langage populaire, les produits de croisements sont appelés chorki, les «mélangés».

populaire, les
44 Valensi 1977.

45 Gallagher 1982

46 Loir 1898; Nicolle 1907. raréfiées; plus près de nous, en Europe, le renard a remplacé le chien là où la rage canine a été contrôlée, et la chauve-souris a tendance à émerger là où la rage vulpine est en voie de disparition ${ }^{47}$.

Tous ces facteurs ont probablement contribué à une profonde modification de l'écologie canine traditionnelle. Le chien va trouver dans le nouveau contexte urbain de la fin du $\mathrm{XIX}^{\mathrm{c}}$ siècle les conditions propices à sa relance démographique. Cette prolifération de l'espèce n'a pu être contrôlée par les mesures précoces mais insuffisantes des pouvoirs publics. Les statistiques issues des campagnes de capture par la police municipale suggèrent un renouvellement démographique rapide: près de 618 chiens errants capturés au dernier trimestre de 1888, 1349 (dont 5 cas de rage) en 1889,2754 en 1906 et 1698 en 1909, rien qu'à Tunis-ville ${ }^{48}$. Le taux de transmission exprimé par l'indice très approximatif du nombre de personnes mordues / nombre d'animaux mordeurs enragés est toujours très supérieur à 1 à Tunis ${ }^{49}$. Les chiens représentent $85 \%$ des cas de morsures animales. La proportion de chiens enragés à propriétaire européen augmente jusqu'à devenir majoritaire vers 1906. De même que l'augmentation des cas de morsures dans les diverses contrées semble suivre la colonisation de peuplement et l'occupation militaire.

La recrudescence de la rage apparaît donc manifestement liée à l'accroissement de la densité canine dans les villes et à l'adoption du chien par une nouvelle catégorie de citadins qui lui procure gîte, eau et nourriture tout en respectant son régime d'errance, ce que l'on admet aujourd'hui être les conditions optimales de reproduction pour l'animal ${ }^{50}$.

\section{Vers une nouvelle pathocénose?}

L'analyse épidémiologique montre que la situation a, jusqu'à aujourd'hui, très peu évolué depuis la fin du XIX ${ }^{\mathrm{e}}$ siècle (fig. 1). L'introduction de la vaccination post-exposition en 1898 a sauvé des vies humaines, mais n'a bien entendu pas changé la tendance épidémique chez le chien. Les études ultérieures désignent toujours le chien errant comme réservoir principal de la maladie ${ }^{51}$, mais la catégorie des chiens «à propriétaire» est elle-même hétérogène et l'appartenance à un propriétaire ne signifie pas surveillance et

47 André-Fontaine/Artois/Ganière 1985

48 L'Echo de Tunis, 1888 et 1889, La Dépêche Tunisienne, 1892, Bulletin municipal de la ville de Tunis, $2^{\mathrm{e}}$ année, Février-Mars 1910,176.

9 Nicolle1906.

Wandeler/Capt 1985

51 Chadli/Zeller/Kchouk 1982 


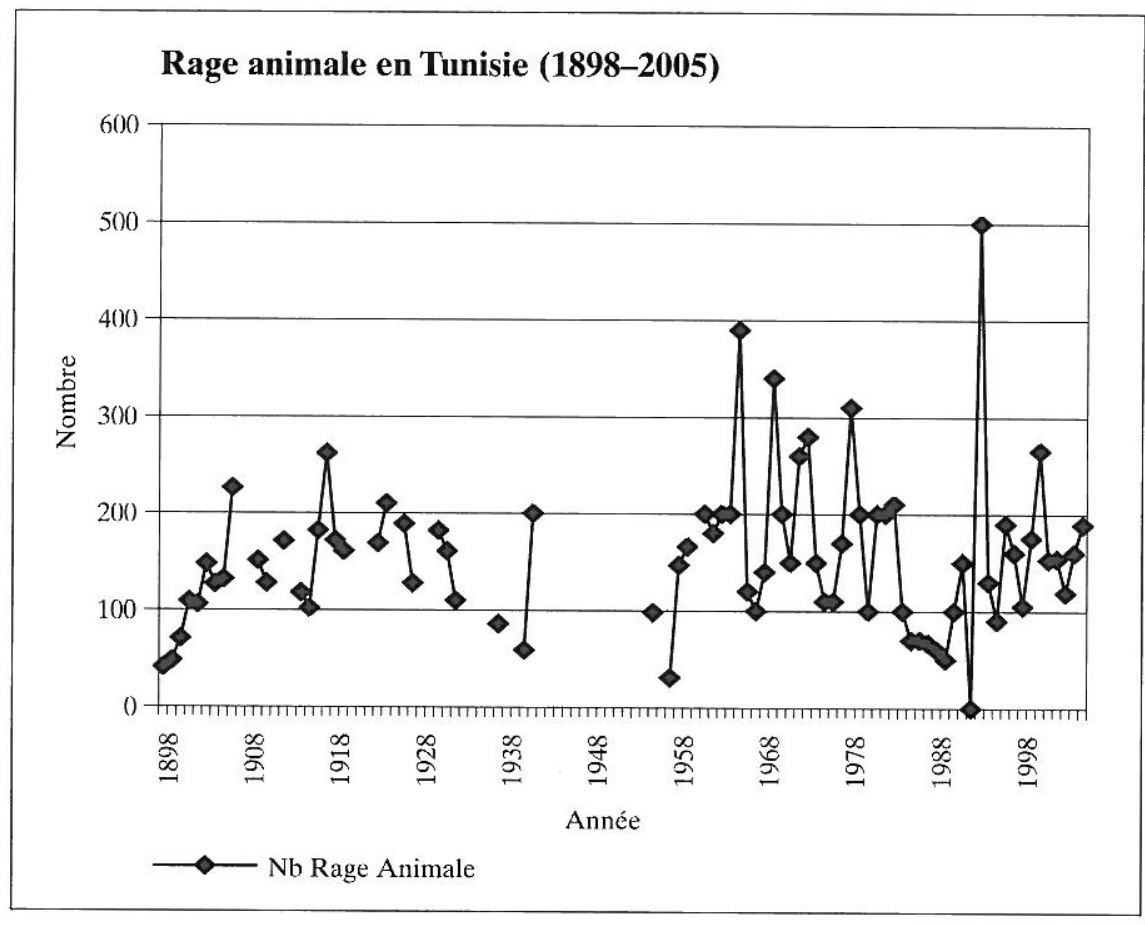

Fig. 1: Evolution de l'incidence de la rage animale (cas confirmés) de 1898 à 2005 Source: Relevé épidémiologique annuel, Archives de l'Institut Pasteur et Ministère de la Santé Publique.

entretien: la plupart des chiens contaminants sont des chiens à propriétaire laissés en divagation péri-domiciliaire. L'habitude du chien de compagnie qui rentre dans les maisons est très peu développée dans les villes. On note aussi sur la courbe de la figure 1 deux pics, l'un à la fin du XIX ${ }^{\mathrm{c}}$ siècle et l'autre qui, paradoxalement, succède à l'application du programme de vaccination systématique du chien entre 1982 et 1987; à peine ce programme fut-il arrêté à partir de 1988 qu'une flambée de rage canine (500 cas) était observée ${ }^{52}$. Ces deux phénomènes n'ont évidemment pas la même signification: le premier pic correspond à un accroissement de la densité canine générale jusqu'à sa stabilisation à un régime de croissance naturel. Le second pic correspond à un accroissement de la population canine «susceptible», incluant les générations de chiens nés après l'arrêt du programme de vaccination.

52 Kharmachi/Hammami 1992
On estime aujourd'hui que les trois quarts des agents pathogènes émergents ou ré-émergents sont des zoonoses dont un tiers est transmissible à l'homme ${ }^{53}$. L'histoire de la rage en Tunisie s'intègre tout à fait dans le cadre général des anthropozoonoses quand la maladie humaine apparaît à la suite de changements significatifs de l'écologie du vecteur et/ou du réservoir. La visibilité de ces maladies dépend souvent de leur passage par l'animal domestique et de la proximité de l'homme. Tout comme la grippe aviaire est aujourd'hui considérée comme «émergente», alors que selon toute vraisemblance elle est ancienne dans certaines régions d'Asie, l'apparition de la rage urbaine en Tunisie au XIX ${ }^{\mathrm{c}}$ siècle fut considérée comme un fait d'importation dans le contexte de l'expansion des empires coloniaux. Charles Nicolle pensait que la conquête de nouveaux territoires avait pour corollaire l'apparition de nouvelles maladies dans des contrées jusque là indemnes, par l'importation de germes auparavant cantonnés dans certaines régions ou par la rupture des équilibres écologiques locaux de divers vecteurs comme les anophèles pour le paludisme ou la mouche tsé-tsé pour la maladie du sommeil ${ }^{54}$. Ces bouleversements, liés à la «civilisation», créaient les conditions favorables pour l'adaptation des pathogènes à de nouveaux hôtes. Ainsi des maladies infectieuses en phase d'extinction pouvaient ressurgir et entamer un nouveau cycle de conservation et transmission. Ceci n'est pas sans nous rappeler les problèmes sanitaires actuels liés à la mondialisation avec l'émergence ou la réémergence de maladies telles que les fièvres hémorragiques dues aux virus Ebola ou la dengue, la fièvre Q, la peste ... La notion d'émergence reste jusqu'à nos jours imprécise: s'agit-il de l'apparition de nouveaux pathogènes? De l'adaptation de pathogènes anciens à l'homme? De l'intrusion d'un pathogène dans un groupe de population jusque là indemne? Dans cette dernière acception, la rage en Tunisie à la fin du $\mathrm{XIX}^{\mathrm{e}}$ siècle pourrait être qualifiée d'émergente mais uniquement pour la population citadine des médina, sinon, du point de vue de la population générale, villes et campagnes confondues, cette étude montre qu'il s'agit d'une intrusion épidémique dans les cités sur un fond endémique probablement très ancien.

La co-évolution de la rage avec des maladies endémo-épidémiques comme l'hydatidose ou la leishmaniose viscérale évoque une pathocénose liée à la multiplication d'un réservoir domestique et son contact plus intime avec l'homme. Le cycle de ces deux dernières maladies est complexe et fait intervenir d'autres couples transmetteurs à l'écologie spécifique: le couple chien/

53 Hansen et al. 2001

54 Nicolle 1937. 
mouton, pour la première et le couple chien/phlébotome, pour la seconde. En fait, les circonstances dans lesquelles ces deux maladies ont «émergé» sont très suggestives. Le kyste hydatique, maladie qui reste longtemps silencieuse sur le plan clinique, a été «révélé» au début du $\mathrm{XX}^{\mathrm{e}}$ siècle par les nouvelles pratiques d'autopsie, de chirurgie et de radiologie introduites par les Européens. Tandis que le kala-azar infantile méditerranéen a été diagnostiqué au départ presque exclusivement chez les enfants italiens, français et maltais, et il a été considéré comme une «maladie nouvelle» et défini comme une catégorie nosologique à part par Charles Nicolle en 1909 avec son réservoir, le chien, identifié en Tunisie la même année.

\section{Conclusion}

En dépit de l'absence des statistiques précises dont se nourrit l'épidémiologie moderne, la documentation historique permet de reconstituer de façon plausible l'histoire de la rage en Tunisie en amont de la date charnière de 1881. Alors que la maladie entame sa phase de déclin en Europe à la fin du XIX ${ }^{c}$ siècle, en Tunisie, elle amorce sa phase ascendante avec un décalage d'un demi-siècle. Les premiers cas, apparus vers 1870 dans la capitale, sont d'emblée mis en relation avec l'arrivée de migrants européens: leur empathie avec le chien parait étrange aux autochtones qui proscrivaient l'animal depuis des siècles. La préexistence probable de cycles enzootiques dans les campagnes sans manifestations épidémiques majeures notifiées, a laissé penser que la maladie était nouvelle dans les villes. Après plus d'un siècle, sa persistance aujourd'hui encore suggère qu'un changement significatif de l'écologie canine s'est opéré à cette période précise et qu'il ne s'agit pas d'importation de virus. La prolifération de chiens errants accompagne (jusqu'à nos jours) l'urbanisation et daterait de cette époque. Cette hypothèse fournit un modèle épidémiologique crédible pour expliquer l'émergence au XIX ${ }^{\mathrm{e}}$ siècle d'anthropozoonoses liées au chien. Elle suggère pratiquement une prophylaxie fondée sur le contrôle démographique de la population canine. L'analyse moléculaire fine de la population virale devrait permettre de dégager les différents variantes en circulation et leurs relations phylogénétiques. En dépit des progrès considérables dans l'épidémiologie et la virologie de l'affection, il reste des inconnues que l'histoire de la médecine met utilement en perspective.

\section{Bibliographie}

Sources primaires

Archives Nationales du Gouvernement de Tunis

Journaux tunisiens: L'Echo de Tunis et La Dépêche Tunisienne

Bulletin municipal de la ville de Tunis

Sources secondaires

Ammar, Sleim, «Ahmed Ibn Al Jazzar et la transmission de son œuvre à l'occident», Tunisie Médicale 75 (1997) 700-704

André-Fontaine G./M. Artois/J.-P. Ganière, «La rage: épidémiologie générale», dans: R. Rosset (éd.), Pasteur et la rage (Paris 1985) 293-301

Babès, Victor, Traité de la rage (Paris 1912)

Balozet, Lucien, «Etat actuel de nos connaissances sur la rage dans les contrées tropicales et subtropicales et sa prophylaxie. La vaccination préventive des chiens», Archives de l'Institut Pasteur de Tunis 4 (1938) 450-459

Bertholon, Henri, «La cynophagie dans l'Afrique du Nord», Bulletin de l'Association Française pour l'Avancement des Sciences (Carthage 1896) 207-208

Bingham, J.,"Canine Rabies Ecology in Southern Africa", Emerging Infectious Diseases 9 (2005) $1337-1340$

Biraben, Jean Noël, «Les maladies en Europe: équilibres et ruptures de la pathocénose», dans: Mirko D. Grmek (éd.), Histoire de la pensée médicale en Occident, t. 1 (Paris 1995)

Bouley, M. H., La rage, moyens d'en éviter les dangers et de prévenir sa propagation (Paris 1870) amussi, Henri, «La rage, son traitement et les insectes vésicants chez les Arabes», Journal Asiatique VIII ${ }^{e}$ série, t. XI et XII (Paris 1888)

- /Ahmed Chérif, «La rage et les insectes vésicants dans la médecine arabe», Archives de l'Institut Pasteur de Tunis 3 (1910) 119-143 et 4 (1910) 165-199

Celse, Traité de médecine, traduction française de Ninin (Paris 1838)

Chabbi, Lahbib, L'imaginaire et la ville: le cas de Tunis au XIX siècle (1850-1881), Thèse de $3^{\mathrm{e}}$ cycle, Université de Sciences Sociales Grenoble 2 (Grenoble 1977)

Chadli A./H. Zeller/M. Kchouk, «Le réservoir du virus rabique en Tunisie», Archives de l'Institut Pasteur de Tunis 1 (1982) 23-31

Chérif, Ahmed, Histoire de la médecine arabe tunisienne, thèse de Médecine soutenue à Bordeaux (Tunis 1908)

Conor, Marthe, «Un texte de Némesien de Carthage sur la pathologie canine (rage et piroplasmose)», Archives de l'Institut Pasteur de Tunis 3 (1912) 131-135

Dumas, Alexandre, Alexandre Dumas à Tunis. Impressions de voyages, présentées, annotées et illustrées par Moncef Charfeddine (Tunis 1982); extrait de «Le Véloce ou Tanger, Alger et Tunis» (première édition, Paris 1848)

Gallagher, Nancy, Medicine and Power in Tunisia: 1780-1900 (Cambridge 1982)

Hansen, G. R. et al., "Emerging Zoonotic Diseases", Emerging Infectious Diseases 7 (2001) 357 Huet Maurice, Le pommier et l'olivier - Charles Nicolle, une biographie (1866-1936) (Paris 1995)

Ibn Jazzar, Zad el Mousafir, édité par Mohamed Snoussi et Radhi Jazi (Carthage 1999)

bn Abî Zayd al Qayrawani, El Risalâ ou épitre sur les éléments du dogme et de la loi de l'islam selon le rite malékite, traduit par Bercher Lucien (Alger 1961)

Kharmachi H./S. Hammami, «Epizootie et zoonoses majeures: évolution de l'enzootie et de l'endémie rabique en Tunisie», Feuillets du Bulletin Epidémiologique et d'Information Vétérinaire, Tunis, 1 (1992) 1

Loir, Adrien, «Les conditions sanitaires et l'hygiène en Tunisie», Revue Générale des Sciences (30 novembre 1896)

«Une épidémie de rage», Bulletin de l'hôpital civil français de Tunis 2 (1898) 49-52

Moulin, Anne Marie, «L'hygiène dans la ville: la médecine ottomane à l'heure pasteurienne (1887-1908)», dans: P. Dumont/F. Georgeon (éds), Les villes ottomanes à la fin de l'Empire (Paris 1992) 186-209 
Nicolle, Charles, «La rage en Tunisie pendant l'année 1906», Archives de l'Institut Pasteur de Tunis 1 (1907) 42-50

«La rage en Tunisie pendant l'année 1914», Archives de l'Institut Pasteur de Tunis 2 (1916) 198-199

- «La question de la rage devant le corps médical tunisien», Tunis Médical (1920) 1-10

- Le destin des maladies infectieuses (Paris 1937)

- Jacques Chaltier, «Quelques faits et quelques expériences concernant la rage», Annales de l'Institut Pasteur 10 (1904) 1-10

Organisation d'Hygiène de la Société des Nations, Rapport sur la Conférence Internationale de la rage, sous la direction de A.-C. Marie, P. Remlinger et H. Vallée (Paris 1927)

Pelis, K., Charles Nicolle, Pasteur's Imperial Missionary: Typhus and Tunesia (Rochester, N.Y. 2006)

Peysonnel, Jean-André, Voyage dans la régence de Tunis (1724) (Tunis 2003)

Remlinger, Paul, «La rareté de la rage à Constantinople», Revue d'hygiène et de police sanitaire 4 (1903) 309-313

Romain, H. Rainero, «La présence italienne en Tunisie de 1868 à 1896 d'après les bulletins diplomatiques et consulaires italiens», Université de Tunis I, Institut Supérieur d'Histoire du Mouvement National, Actes du colloque Les relations tuniso-italiennes dans le contexte du Mouvement National, Actes du

protectorat (Tunis 1999) 19-30
Sebag, Paul, Tunis, histoire d'une ville (Paris 1989)

Valensi, Lucette, Fellahs tunisiens: l'économie rurale et la vie dans les campagnes aux $18^{\circ} \mathrm{et}$ $19^{\mathrm{c}}$ siècles (Paris 1977)

Wandeler, A. I/S. Capt, «Ecologie du chien», dans: R. Rosset (éd.), Pasteur et la rage (Paris 1985) $115-120$ 